\title{
Las consecuencias actuales de la privación ilícita de obras de arte en tiempos del nazismo y la inmunidad del Estado: el caso Cassirer $^{1}$
}

\section{The current consequences of the illegal deprivation of art works in the Nazism time and State immunity: the Cassirer case}

\section{Soledad Torrecuadrada García-Lozano}

s.torrecuadrada@uam.es

Profesora Titular de Derecho Internacional Público en la Universidad Autónoma de Madrid. Acreditada a Catedrática por resolución del Ministerio de Educación, Cultura y Deporte de 2 de julio de 2012. Doctora en Derecho (1996) por la Universidad Autónoma de Madrid, Diploma del Center for Studies and Research de la Academia de Derecho Internacional de La Haya (1996) y M.A. en Relaciones Internacionales por el IUIOG, adscrito a la Universidad Complutense de Madrid (1992).

Resumen: Hay consecuencias del Holocausto que aún no se han resuelto. Buena muestra de ello son las reclamaciones de devolución de los bienes que fueron incautados por los nazis, especialmente obras de arte, que llegan hasta nuestros días. La inexistencia de órganos internacionales competentes para resolver aquellas junto con la carencia de normas internacionales aplicables a los supuestos acaecidos antes de su entrada en vigor nos ubican en un escenario de soluciones heterogéneas, debido a la dispersión en lo que se refiere a las normas nacionales existentes al efecto. Por otra parte, los tribunales estadounidenses, que han tenido que resolver múltiples demandas en este sentido, han aplicado sistemáticamente la excepción de expropiación, que presenta dificultades en lo que a su compatibilidad con las normas internacionales (en la actualidad de naturaleza consuetudinaria) existentes sobre las inmunidades de los Estados y de sus bienes, así como sobre la jurisprudencia de la Corte Internacional de Justicia existente en este punto.

Palabras claves: obras de arte, nazismo, inmunidad de jurisdicción, inmunidad de ejecución, Derecho Internacional Público.

\begin{abstract}
There are consequences of the Holocaust that remain unsolved. We can think, for example, of the claims for the restitution of the properties that were seized by the Nazis, especially art pieces that still have not been resolved. The absence of competent international organs to resolve these cases, along with the scarce international norms that are effectively applicable to events happening before their entry into force, brings us to a diverse scene marked by the heterogeneity of national legislations and national courts rulings. On the other side, American
\end{abstract}

1 Artículo enviado el 16.03.2018 y aceptado el 26.07.2018.

Número de página no utilizable para citar 
Las consecuencias actuales de la privación ilícita de obras de arte en tiempos del nazismo Soledad Torrecuadrada García-Lozano

courts have ruled several times on these matters, systematically leaving aside the exception of expropriation. These decisions arise compatibility issues with the existing set of international norms - which has nowadays a customary nature- about the immunity of the States and their properties, as well as with the most recent case law of the International Court of Justice on this issue.

Key words: Art Works, Nazism, Jurisdictional Immunity, Immunity from Execution, Public International Law.

\section{Introducción}

El Holocausto fue un crimen internacional de extraordinaria gravedad, cuyas consecuencias perduran cuando han transcurrido ya más de ochenta años desde las Leyes de Núremberg. Unas de esas consecuencias, que en la actualidad están sin resolverse totalmente, son las derivadas de las privaciones ilícitas de obras de arte a coleccionistas judíos, en lo que fue un instrumento de la política genocida del nazismo, consistente no solo en privar a las personas perseguidas de sus fuentes de ingresos para subsistir, sino también de cualquier bien económicamente cuantificable. Muy especialmente, como indica D. Brown, parte de la deshumanización internacional de los judíos consistió en separarlos primero de sus propiedades².

No podemos olvidar que se calcula que las pérdidas de obras de arte producidas durante el régimen nazi en Europa ascendieron a veinte billones y medio de dólares ${ }^{3}$, en lo que fue el mayor robo de la historia ${ }^{4}$. Evidentemente, se trata de un dato aproximado que deriva de la consideración de que más de 240.000 obras de arte fueron desplazadas, perdidas o destruidas ${ }^{5}$. Las pertenecientes a la primera categoría (desplazadas) fueron expoliadas con el propósito de

2 Vid. D. Brown, "Litigating the Holocaust: A Consistent Theory in Tort for the Private Enforcement of Human Rights Violations", Pepperdine Law Review, 27 (2000): 560.

3 Aportan este dato, entre otros, K. D. Walton, "Leave No Stone Unturned: The Search for Art Stolen by the Nazis and the Legal Rules Governing Restitution of Stolen Art", Fordham Intellectual Property, Media and Entertainment Law Journal, 9(2) (1999): 552); E.A. Maples, "Holocaust Art: It Isn't Always Finders Keepers, Losers Weepers: A Look at Art Stolen during the Third Reich", Tulsa Journal of Comparative and International Law, 9 (1) (2001): 356, y S. Choi, "The Legal Landscape of the International Art Market After Republic of Austria v. Altmann”, Northwestern Journal of International Law \& Business, 26(1) (2005): 167.

4 Así lo denomina Brown, "Litigating the Holocaust", 560.

5 Me refiero al expolio que existió al amparo del nazismo no solo en Alemania. Pueden verse trabajos en los que se estudia el expolio en otros lugares, como el de G. Aalders, "Pillage y (non) restitution des oeuvres d'art aux Pays-Bas (19402001)", Revue d'Histoire de la Shoah, (186) (2007): 195. También, a modo de ejemplo, el Informe Matteoli, "Rapport au Premier ministre de la Mission d'étude sur la spoliation des Juifs de France", http://www.civs.gouv.fr/images/pdf/matteoli/Rapport_Matteoli_1997.pdf [consulta 30.jul.2018]. Pueden verse otros informes sobre el expolio en Francia en la página de la Commission pour l'indemnisation des victimes de spoliations, http://www.civs.gouv.fr/fr/ressources-documentaires/la-mission-matteoli/ [consulta 30.jul.2018].

Número de página no utilizable para citar 
cumplir el deseo de Hitler crear un Museo en Linz, su ciudad natal, con obras que consideraba ejemplos de "arte y cultura superior"; ; en un edificio para el que él mismo había preparado los planos y que se concebía como un monumento al nazismo ${ }^{7}$. Entre las perdidas o destruidas hubo cierta confusión, debido a que algunas de ellas se creyeron destruidas en un primer momento, por pertenecer a la categoría de "arte degenerado" (en la que se ubicaba el arte moderno o abstracto entre otros que el nazismo calificaba como productos inferiores), pero pudimos afortunadamente comprobar que no fue así, debido a su aparición posterior ${ }^{8}$.

Con ello se advierte que las razones económicas, en algunos casos, pesaron más que la obediencia en aquel período histórico ${ }^{9}$. Los avances técnicos han producido la proximidad de los catálogos de los museos, que podemos contemplar desde nuestra casa y han permitido identificar las obras que fueron ilícitamente privadas a sus propietarios, dando lugar a una relación directamente proporcional entre el número de apariciones y las reclamaciones de restitución.

Uno de los problemas con los que nos encontramos en la actualidad es que no existen órganos internacionales a los que acudir cuando un descendiente de la víctima de la privación identifica la obra de su antepasado, por lo que la solución que les queda es instar su devolución ante los órganos creados al efecto en algunos Estados (como Francia, Austria o Alemania) y, en su defecto a los tribunales nacionales. Esto ha provocado una suerte de heterogeneidad en lo que a la aplicación de procedimientos se refiere y, en la medida en que las legislaciones estatales resultan dispares en esta materia, evidentemente también en las soluciones alcanzadas.

En estas páginas me referiré, en un primer lugar, a la ausencia de instrumentos de Derecho Internacional Público que permitan resolver, con un mínimo de homogeneidad, las situaciones en presencia, para en un momento posterior centrarme a la aplicación (o no) de normas del mismo ordenamiento relativas a la inmunidad de jurisdicción del Estado extranjero, especialmente a la luz de la denominada excepción de expropiación a la inmunidad de jurisdicción que se incorpora en la Ley estadounidense de inmunidad, que no encuentra fundamento en la Convención de Naciones Unidas sobre las inmunidades de los Estados y de sus bienes de 2004 (en adelante, Convención de 2004), así como a la lectura que de la inmunidad de ejecución ha realizado la Corte Internacional de Justicia en el asunto Alemania c. Italia.

\footnotetext{
S. Choi, “The Legal Landscape”, 168.

Vid. M.I. Turner, “The Innocent Buyer of Art Looted during World War II”, Vanderbilt Journal of Transnational Law, (32) (1999): 1514.

8 Aunque ciertamente, muchos ejemplos de ese arte fueron quemados. Ver en este sentid Turner, “The Innocent Buyer”, 1515.

9 Lo que ha hecho que Brown, "Litigating the Holocaust", 557, traduzca las reflexiones de N. M. Sher; M. Berenbaum; A. L. Smith y B. Neuborne "The Search For Nazi Assets: A Historical Perspective”, Whittier Law Review, 20 (1998): 7, en la siguiente afirmación: "Historically speaking, genocide is a very lucrative business". Aunque debe considerarse que en la obra citada en último lugar no solo se reflexiona sobre los bienes artísticos, sino acerca de la generalidad de lo expoliado. 


\section{La inexistencia de normas internacionales en relación con la restitución de los bienes expoliados por los nazis}

La solución del expolio producido en la Segunda Guerra Mundial es muy complicada debido a la confluencia de múltiples factores, entre otros: la víctima de la privación de las obras artísticas, en el mejor de los casos, ya no será residente en el mismo lugar que entonces, incluso tendrá una nacionalidad diferente de la que poseía cuando se produjo el hecho ilícito ${ }^{10}$; los bienes que fueron confiscados pueden no encontrarse en el territorio en el que se produjo aquella apropiación ilícita o la venta a bajo precio; por último, puede haber sido objeto de compra-ventas subsiguientes, con lo que a quien se reclama su entrega puede ser un adquirente de buena fe, que le convierte en víctima de la solución a la ilicitud previa. Las alteraciones producidas en los requisitos de la protección diplomática convierten esta institución en impracticable como instrumento para los fines que perseguimos, especialmente porque no se trata de exigir la responsabilidad del causante del daño, sino de procurar la devolución del bien por el poseedor actual, lo que excede (cuando no existe coincidencia entre este último con aquel a quien se atribuye el ilícito) del alcance de la institución considerada.

Además, carecemos de normas internacionales que permitan resolver las reparaciones derivadas del expolio nazi ${ }^{11}$. Es cierto que, las potencias aliadas, antes de concluir la Segunda Guerra Mundial, adoptaron el 5 de enero de 1943 la Declaración Conjunta de Londres, en la que negaban el efecto de las confiscaciones directas e indirectas en los territorios ocupados. Texto que, de contar con efectos jurídicamente vinculantes, los tendría exclusivamente para los Estados que participaron en su adopción. Por su parte, el Acta Final de la Conferencia de Bretton Woods, incorporaba una Resolución (la Sexta) en la que, se recomendaba a los Estados representados en ella que instasen a los países neutrales a evitar cualquier transferencia de activos, entre otros, de objetos de arte, provenientes de los territorios ocupados, con independencia de su naturaleza pública o privada.

Es todo con lo que contábamos hasta 1998 cuando se adoptó la Declaración de Washington. Desde entonces se han producido algunas declaraciones de naturaleza igualmente política, centradas en la necesidad de resolver la situación derivada del expolio nazi. En el caso de la

10 Nos encontraríamos ante un cambio de nacionalidad que no puede calificarse de involuntario, puesto que si bien las Leyes de Núremberg privaron a las personas judías de la ciudadanía alemana, la adquisición posterior de una nacionalidad concreta sí que resultó voluntaria. Quiero con esto decir que no es comparable a los supuestos claramente reconocidos al efecto, como son los de disolución o desmembramiento de Estados, por ejemplo.

11 W. Sandholtz, en "Plunder, Restitution, and International Law", International Journal of Cultural Property, 17 (2010): 146 y ss., hace un recorrido por la evolución de las normas en este punto, remontándose a su emergencia de las normas antisaqueo, recordando en este punto el saqueo napoleónico. Sobre los trabajos de los aliados para procurar la restitución de bienes culturales, vid. M.J. Kurtz, “The Allied Struggle over Cultural Restitution, 1942-1947”, International Journal of Cultural Property, 17 (2010): 177 y ss. 
Declaración de Washington, su redacción comienza recordando que los Estados actúan "en el desarrollo de un consenso sobre principios no vinculantes para asistir en la resolución de problemas relativos al arte confiscado por los nazis"12, aclarando así sus efectos. En ella, los Estados acuerdan destinar recursos para identificar las obras de arte confiscadas por los nazis que no hayan sido restituidas. Si se identificase a los propietarios de obras de arte confiscadas por los nazis, encontradas y no restituidas deberían adoptarse medidas rápidamente para lograr una solución justa y equitativa, aunque reconociendo las reclamaciones tendrán que analizarse particularmente, teniendo en cuenta los hechos y circunstancias que pueden caracterizar un caso concreto.

Poco después, en el seno del Consejo de Europa, su Asamblea Parlamentaria adoptó la Resolución $1205^{13}$ que recuerda las modalidades de reparación de un hecho ilícito desde la perspectiva de la responsabilidad internacional (restitución o en su defecto, indemnización). Al año siguiente de adoptarse esta Resolución se inicia el Foro Internacional de Vilna (el 5 de octubre de 2000) bajo los auspicios de la misma organización para dar seguimiento a la Declaración de Washington. Fruto de la convocatoria se adopta una nueva Declaración, la de Vilna, que insta a los gobiernos, museos, marchantes de arte y otros agentes relevantes proporcionar toda la información necesaria para la restitución ${ }^{14}$. La información solicitada ha de ser pública y accesible, para que pueda lograrse el propósito perseguido y ha de compartirse para su mayor difusión. Además, reconoce la necesidad urgente de trabajar en maneras de lograr una solución justa y equitativa para el problema del arte expoliado por los nazis cuando no se pueda identificar a sus propietarios o herederos ${ }^{15}$.

Casi nueve años después de Vilna, el 30 de junio de 2009, se adoptó la Declaración de Terezín, en la que una vez más, se incluye la mención a su naturaleza no normativa, puesto que los declarantes la formulan "teniendo presente la naturaleza jurídicamente no vinculante de esta Declaración y, consiguientemente, las responsabilidades morales, sin perjuicio del Derecho Internacional y obligaciones aplicables" ${ }^{\prime 6}$. Además de lo anterior, se afirma la necesidad de resolver de forma efectiva las cuestiones relativas a las propiedades de las que se privó ilicitamente a las víctimas del Holocausto, procurando una solución justa y equitativa de acuerdo con el ordenamiento interno (alentando a los Estados a su modificación para evitar dificultades

12 Traducción de la autora. El texto original en inglés dice "In developing a consensus on non-binding principles to assist in resolving issues relating to Nazi-confiscated art". El texto completo puede encontrarse en "Washington Conference Principles on Nazi-Confiscated Art. 3 December 1998”, http://www.lootedart.com/MG7QA043892 [consulta 30.jul.2018].

13 La resolución 1205 (1999), relativa a los bienes culturales expoliados a los judíos, adoptada el 4 de noviembre de 1999.

14 Vid. Declaración íntegra en "Vilnius Forum Declaration. 5 octiber 2000", http://www.lootedart.com/MFV7EE39608 [consulta 30.jul.2018].

15 "Vilnius Forum Declaration".

16 Traducción propia. El texto original en inglés dice: "Keeping in mind the legally non-binding nature of this Declaration and moral responsibilities thereof, and without prejudice to applicable international law and obligations". Vid. "Terezin Declaration. 30 June 2009”, http://www.lootedart.com/NPMG2Q663241 [consulta 30.jul.2018]. 
Las consecuencias actuales de la privación ilícita de obras de arte en tiempos del nazismo Soledad Torrecuadrada García-Lozano

e impedimentos al propósito perseguido) e internacional, siempre considerando todos los elementos presentes en cada uno de los supuestos.

Las Declaraciones hasta aquí referidas poseen exclusivamente un valor político. Así lo ha recordado un tribunal estadounidense en el caso Dunbar Seger-Thomschitz, cuyo origen se encuentra en la posesión actual por parte del Sr. Dunbar de un cuadro del pintor expresionista austriaco Oskar Kokoschka denominado "Retrato de Juventud". La Sra Seger-Thomschitz había reclamado la restitución de la obra por entender que fue confiscada por los nazis a la familia de su esposo. Ciertamente, el suegro de la reclamante vendió la pintura en cuestión junto con otras cuatro a un marchante de arte judío presunto colaborador de los nazis. Cuando la madre del propietario actual adquirió en 1946 la pintura, no investigó el origen de esa venta. El Tribunal de Distrito que resolvió el caso entendió que el legítimo propietario es el Sr. Dubar que adquirió la propiedad de la pintura de su madre por herencia en 1973. La recurrente, Sra. Seger-Tomscitz solicitó entonces al Tribunal de Apelaciones la inaplicación de la legislación de Luisiana y su sustitución por la "Declaración de Terezin", de 2009. Cuestión a la que el Tribunal no pudo sino responder negativamente, debido a la naturaleza política, que no jurídica del acto en cuestión ${ }^{17}$.

Si bien, carecemos de normas internacionales para resolver los supuestos en presencia, para los conflictos originados más recientemente contamos con los tratados internacionales auspiciados por la Unesco de 1970 sobre las medidas que deben adoptarse para prohibir e impedir la importación, la exportación y la transferencia de propiedad ilícitas de bienes culturales y el de UNIDROIT de 1995 sobre restitución de objetos culturales robados o ilícitamente exportados, que resultan de aplicación para las situaciones posteriores a su entrada en vigor. A pesar de no resultar aplicables a los hechos como los que nos ocupan, podrían inspirar las soluciones que los órganos nacionales aporten a los casos que se les presenten cuyo origen sea anterior su vigencia. Interpretación a la luz de las normas internacionales que no resulta exigible jurídicamente, pero cuya aplicación vendría avalada por motivos de coherencia y razonabilidad.

\section{Aspectos relacionados con el Derecho Internacional Público en estas reclamaciones}

La primera cuestión que han de resolver las víctimas del expolio nazi cuando se encuentran en alguna colección pública o privada el cuadro que les fue arrebatado es cómo actuar para intentar recuperarlo. Como vimos antes, en ausencia de órganos internacional a los que acudir, desde una perspectiva de Derecho Internacional Público procedería dirigirse al Estado (o al sucesor o

17 Vid. "La decisión de la Corte de apelaciones de Estados Unidos, Quinto circuito, de 20 de agosto de 2010", http://www.ca5.uscourts.gov/opinions/pub/09/09-30717-CV0.wpd.pdf [consulta 30.jul.2018].

Número de página no utilizable para citar 
continuador de su personalidad jurídica) que se apropió del bien para procurar su restitución. Ello con el propósito de que sea él quien reclame al actual poseedor del bien, compensándole por la pérdida que es, en definitiva, una solución inspirada, entre otros, en el artículo 7.ii) de la Convención sobre medidas que deben adoptarse para prohibir e impedir la importación, la exportación y la transferencia de propiedad ilícita de bienes culturales de 1970.

Sin embargo, la práctica no ha seguido ese camino, pues las víctimas y sus herederos han preferido en muchos casos acudir a los tribunales nacionales de su lugar de residencia y, en la mayoría (o al menos en los más mediáticos), han sido los tribunales estadounidenses los que han tenido que resolver estas cuestiones.

\subsection{La inmunidad de jurisdicción del Estado extranjero. La excepción de expropiación}

En relación con la competencia judicial, uno de los primeros elementos a considerar es la extraterritorialidad de los tribunales del Estado. En los casos en presencia, pensemos que el demandante acude a los tribunales de su residencia (Cassirer, Altmann, Alsdorf v. Benninson, Seger-Thomschitz, entre otros) para que conozca de hechos que se produjeron extraterritorialmente, puesto que nos encontramos ante la demanda de restitución de un bien del que se privó a la familia de aquel en el que fuera territorio alemán hasta 1945.

En todo caso, cuando la obra de arte se encuentra en manos de un Estado el reclamante se encontrará con la barrera que supone la inmunidad de jurisdicción primero y, de obtener una sentencia negativa a los intereses del Estado, la posterior inmunidad de ejecución, con las que las normas de Derecho Internacional protegen a los sujetos de este ordenamiento. En el caso Cassirer c. la Fundación Colección Thyssen-Bornemisza y el Reino de España, la pintura "Rue Saint-Honoré, après-midi, effet de pluie”, que pintara Camille Pisarro en 1897 es propiedad del Reino de España ${ }^{18}$, se interpuso una demanda en San Francisco. España adujo dos motivos para su desestimación: en primer lugar la ausencia de competencia material por parte del tribunal para conocer del fondo del asunto debido a la inmunidad de jurisdicción de la que goza el Estado

18 Del comportamiento de Claude Cassirer (nieto de Lilly, que tuvo que vender el cuadro a bajo precio en la Alemania nazi) se desprende este reconocimiento, pues se dirigió al Ministerio de Cultura en 2000 solicitando la restitución del cuadro que había pertenecido a su abuela hasta 1939. La misma evidencia se desprende de la reclamación de los cinco congresistas referidos antes, así como por parte de Estados Unidos, si hacemos caso de los documentos que se publicaron de Wikileaks, la Embajada de Estados Unidos habría ofrecido resolver el conflicto respecto del tesoro capturado ilícitamente por la empresa Odyssey por el cuadro que ahora nos ocupa (Reuters, "EEUU quiso cambiar el tesoro del Odyssey por un cuadro-Wikileaks, http://es.reuters.com/article/entertainmentNews/idESMAE6B80DU20101209 [consulta 30.jul.2018]). Reconocimiento innecesario, por otra parte, puesto que España había adquirido la colección permanente del Barón Thyssen Bornemisza, compuesta por 775 obras, entre las que se encontraba la de Camille Cassirer posteriormente reclamada, a cambio de 338.216.958 dólares, íntegramente sufragados por el Estado español. Así se establece en el "Real Decreto-ley 11/1993, de 18 de junio, sobre medidas reguladoras del contrato de adquisición de la colección ThyssenBornemisza", https://www.boe.es/diario_boe/txt.php?id=BOE-A-1993-15901 [consulta 30.jul.2018]. El Real Decreto Ley 11/1993 establece la cantidad en pesetas, que ascendía a un total de 42.277.120.000 (artículo 1). 
Las consecuencias actuales de la privación ilícita de obras de arte en tiempos del nazismo

Soledad Torrecuadrada García-Lozano

español propietario de la obra reclamada ${ }^{19}$; y, en segundo lugar, la ausencia de objeto, debido a que por una parte, el Estado español no es el causante de la privación y por otro que la compensación percibida por la Sra. Cassirer había extinguido la responsabilidad internacional. Aquí nos centraremos solo en el primero.

De acuerdo con la Convención de Naciones Unidas sobre las inmunidades de los Estados y de sus bienes de 2004, si bien los Estados gozan de inmunidad ante los tribunales del foro, esta inmunidad no es absoluta, sino que, si el origen de la controversia se encuentra en uno de los "procesos en los que la inmunidad no se puede hacer valer", dado que el Estado se ha comportado como podría hacerlo un particular, la excepción de inmunidad de jurisdicción no sería predicable. En este punto, no parece plausible ubicar el comportamiento en cuestión (que, en definitiva, es la adquisición del cuadro por parte de España al Barón Thyssen-Bornemisza, junto con el resto de su colección) dentro del alcance de ninguna de las excepciones a la inmunidad del Estado. Lo más próximo que podemos encontrar en el citado texto convencional es su artículo 13, referido a "la propiedad, la posesión y el uso de bienes" ${ }^{20}$, que carece de utilidad en este sentido puesto que el objeto de la demanda no versa sobre un derecho o interés derivado de "sucesión, donación u ocupación de bien vacante", dado que fue una compraventa de la colección pictórica por parte del Estado, y tampoco nos referimos a bienes en "fideicomiso, bienes integrantes de la masa de la quiebra o bienes de una sociedad en caso de disolución", que son las situaciones en las que no resulta aplicable la inmunidad de jurisdicción del Estado extranjero por este concepto. Desde el punto de vista del Derecho Internacional Público, en consecuencia, el Estado español gozaría de inmunidad de jurisdicción en el caso en presencia.

Sin embargo, la Ley estadounidense sobre la inmunidad del Estado extranjero (FSIA), incorpora una excepción añadida a la relación de la Convención de Naciones Unidas sobre las inmunidades de los Estados y de sus bienes de 2004, que es la que han considerado los tribunales estadounidenses, cuya interpretación sirvió de fundamento para conocer del fondo del asunto. En este sentido la Sección 1605 (A) (3), contiene la conocida como excepción de expropiación, que excluye toda la inmunidad en relación con aquellas reclamaciones originadas por la adquisición de los derechos de propiedad en violación del Derecho Internacional ${ }^{21}$. Para que se

19 En virtud de 28 U.S. Code $\$ 1604$ - Immunity of a Foreign State from Jurisdiction. Sobre los problemas que presenta la norma en cuestión y la jurisprudencia que la interpreta, puede verse, entre otros: L. Pyke Malson, K. Nesbitt, A. Portnoy y B. Kurtz "The Foreign Sovereign Immunities Act: 2009 Year in Review", Law and Business Review of the Americas, 17 (2011): 39 y ss.

20 El precepto en cuestión establece que no se podrá hacer valer la inmunidad, por lo que se refiere a la propiedad, posesión y uso de bienes "en un proceso relativo a la determinación de: a) un derecho o interés del Estado respecto de bienes inmuebles situados en el Estado del foro, la posesión o el uso por el Estado de esos bienes inmuebles o una obligación del Estado nacida de su derecho o interés respecto de tales bienes inmuebles o de su posesión o uso de esos bienes; b) un derecho o interés del Estado respecto de bienes muebles o inmuebles, nacido en virtud de sucesión, donación u ocupación de bien vacante; o c) un derecho o interés del Estado respecto de la administración de bienes, tales como bienes en fideicomiso, bienes integrantes de la masa de la quiebra o bienes de una sociedad en caso de disolución." Sin que el caso en presencia se encuentre en ninguna de las excepciones indicadas al efecto.

21 La redacción de la excepción en cuestión es la siguiente: "in which rights in property taken in violation of international law are in issue and that property or any property exchanged for such property is present in the United States in connection with a commercial activity carried on in the United States by the foreign state; or that property or any property exchanged 
observe esta excepción es necesario (además de la ilicitud de la adquisición recién referida) que se cumplan los requisitos indicados en la misma sección: la acción en presencia ha de encontrar fundamento en una reclamación a un gobierno de una obra propiedad de un organismo estatal o gestionada por él; y la realización de actividades comerciales en Estados Unidos por parte del citado organismo.

A pesar de que Alemania y no España fue responsable de la privación, los tribunales estadounidenses se declararon competentes para conocer del fondo del asunto, interpretando alguno de los requisitos recién mencionados de forma flexible. Así, la norma establece como presupuesto para su aplicación la adquisición ilícita del bien, pero no que la ilicitud sea atribuible al Estado al que se está reclamando aquel. En definitiva, el hecho relevante es que se haya producido la ilicitud, no quién ha sido su causante, con lo que nos alejamos de las estructuras básicas de la responsabilidad, en las que quien ha de reparar es precisamente el causante del daño ${ }^{22}$.

Lo anterior implica que es el tercero adquirente de buena fe quien ha de reparar a la víctima de la ilicitud ajena, ya sea entregando el bien o compensando económicamente para conservar su posesión, lo que resulta ciertamente incomprensible desde la perspectiva de la responsabilidad. Cabe plantear que, si el particular fue ilícitamente privado del bien, las transmisiones posteriores quedan igualmente viciadas por este motivo. Sin embargo, como afirma P. O'Keefe, en los sistemas jurídicos de derecho romano, un adquirente de buena fe pasado el tiempo establecido en la Ley, adquiere un título incontestable, aunque se trate de un bien que originariamente fue robado $^{23}$. Por el contrario, en los sistemas de Common Law, como el Reino Unido, la adquisición ilícita priva de la posibilidad de adquirir el título, pero el adquirente de buena fe transcurrido un

for such property is owned or operated by an agency or instrumentality of the foreign state and that agency or instrumentality is engaged in a commercial activity in the United States".

22 Vid. Sentencia de 8 de septiembre de 2009, del Tribunal de apelaciones, Noveno circuito, Claude Cassirer v. Kingdom of Spain, a Foreign State, http:// caselaw.findlaw.com/us.9th-circuit/1116366.html [consulta 30.jul.2018].

23 Vid. Informe presentado por P. O'Keefe, “Annexe 2. Document de travail sur les aspects juridiques”, en Doc. 8563 révisé (2 de noviembre de 1999). Rapport. Biens culturels des juifs spoliés. Assemblée parlementaire Conseil de l'Europe, en https://assembly.coe.int/nw/xml/XRef/X2H-Xref-ViewHTML.asp?FileID=8759\&lang=fr [consulta 30.jul.2018]. La idea del texto en p. 19. 
Las consecuencias actuales de la privación ilícita de obras de arte en tiempos del nazismo

Soledad Torrecuadrada García-Lozano

tiempo de posesión pacífica sí que lo hace ${ }^{24}$; y en Estados Unidos la legislación varía en función del Estado en el que nos encontremos ${ }^{25}$.

En relación con los requisitos que establece la norma estadounidense para su aplicación, es evidente que, si se reconoce a la Fundación Colección Thyssen como agencia instrumental del Estado, está claro que se ha presentado la reclamación de restitución de una obra propiedad de aquella agencia instrumental o por ella gestionada, cumpliéndose así el primero de los establecidos en la norma. Sin embargo, la apreciación de la presencia del segundo (la realización de actividades comerciales en Estados Unidos por parte del citado organismo) ${ }^{26}$, imprescindible para la aplicación de la excepción de expropiación, es interpretada por el Tribunal de forma extensa debido a que, para identificarla, no es necesaria una actividad comercial continua y sistemática ${ }^{27}$. El mismo tribunal se refiere al caso Altmann, en cuya sentencia se concluyó que la autoría, promoción y distribución de libros y otras publicaciones en los Estados Unidos para explotar cuadros expropiados era suficiente para constituir "actividad comercial" a los efectos de aplicación de la FSIA ${ }^{28}$.

Aplicando este criterio, el tribunal concluye que la Fundación Colección Thyssen-Bornemisza (creada para la exhibición de la colección adquirida por el Estado) desarrolla una actividad comercial suficiente en el territorio de Estados Unidos, pues se pueden adquirir libros, posters, reproducciones autorizadas de obras de arte, entradas on line o productos de la tienda del museo por el mismo medio, destacándose entre ellos la venta a residentes en Estados Unidos de reproducciones de la obra de Pissarro objeto de la reclamación, así como de otros productos

24 En el Reino Unido se adoptó en 2009 la Holocaust (Stolen Art) Restitution Bill, que tiene por objeto "Provide for the transfer from public museum and gallery collections of arts, artefacts and other objects stolen between 1933 and 1945 by or on behalf of the Nazi regime, its members and sympathisers; to provide for the return of such artefacts and objects to the lawful owners, their heirs and successors; and for connected purposes." Vid. texto íntegro en "Holocaust (Stolen Art) Restitution Bill”, https://publications.parliament.uk/pa/cm200809/cmbills/035/2009035.pdf [consulta 30.jul.2018]. Debe considerarase que el informe es de 1999 y que las legislaciones estatales de los Estados principalmente afectados por el expolio han sido modificadas con posterioridad a esa fecha para permitir la restitución en el supuesto concreto que nos ocupa.

25 Vid. Informe presentado por P. O'Keefe. Sobre la situación del derecho español en este punto, aplicado al caso Cassirer, vid. B. Arp, "Dos males, un bien no hacen: el asunto Cassirer ante los tribunales estadounidenses y la inmunidad de jurisdicción de España”, Revista Española de Derecho Internacional, LXIII (2) (2011): 161 y ss., y C.M. Díez Soto “Cassirer v. Fundación Thyssen: adquisición por usucapión extraordinaria de obra de arte robada durante el Holocausto", Cuadernos de Derecho Transnacional, 8(2) (2016): 377 y ss.

26 Sobre la excepción comercial puede verse el estudio clásico de J.E. Donoghue, “Taking the 'Sovereign' Out of the Foreign Sovereign Immunities Act: A Functional Approach to the Commercial Activity”, Yale Journal of International Law, 12 (2) (1992): 490 y ss, en http://digitalcommons.law.yale.edu/cgi/viewcontent.cgi?article=1599\&context=yjil [consulta 30.jul.2018].

27 Según la definición que incorpora la misma norma a estos efectos “"commercial activity' means either a regular course of commercial conduct or a particular commercial transaction or act. The commercial character of an activity shall be determined by reference to the nature of the course of conduct or particular transaction or act, rather than by reference to its purpose".

28 Vid. Sentencia de 12 de diciembre de 2002, en el caso Altmann v. Republic of Austria, Nos. 01-56003, 01-56398, 317 F.3d 954 (2002), https://www.leagle.com/decision/20021271317f3d95411167 [consulta 30.jul.2018].

Número de página no utilizable para citar 
relacionados con ella ${ }^{29}$. Se acredita de este modo la existencia de actividad comercial, motivo por el cual, se declaran cumplidos los dos requisitos establecidos para la aplicación de esta excepción a la inmunidad.

Desde una perspectiva de Derecho Internacional Público y, más concretamente, de la inmunidad de jurisdicción, la existencia de actividad comercial, permitiría a los tribunales del foro conocer de las eventuales reclamaciones que se originaran como consecuencia de la citada transacción mercantil (artículo 10 de la Convención de Naciones Unidas sobre las inmunidades de los Estados y de sus bienes de 2004), lo que queda al margen de la aplicación de la excepción de inmunidad es la actividad comercial en sí misma (cuando el objeto de la demanda sea la venta on line, la introducción de anuncios en prensa, etc.). Con este mismo enfoque, la existencia de relaciones comerciales no es un elemento a tener en cuenta para aplicar una excepción a la inmunidad de jurisdicción a relaciones distintas de la misma transacción afectada. En todo caso, convengamos que, en este punto, la flexibilidad a la hora de ponderar la presencia del requisito en cuestión ha sido amplia.

Por lo demás, la excepción contenida en la Sección 1605 (A) (3) de la Ley estadounidense de inmunidades es una excepción que está permitiendo a sus tribunales entrar a conocer del fondo de las reclamaciones suscitadas en relación con los bienes expoliados en tiempos del nazismo. Así, volviendo al caso Altmann, el sentido de la decisión del tribunal californiano que conocía del caso fue idéntica a la producida en Cassirer y por el mismo motivo (la excepción de expropiación), aunque entre los dos supuestos había una diferencia sustantiva: en aquel el causante de la privación ilícita fue el Estado austriaco que poseía las obras de Klimt (que se encontraban en la Galería Belvedere de Viena) y, en cuanto tal, el responsable. Situación muy diferente de la que contextualiza el caso Cassirer, en la que el Reino de España no tuvo relación alguna con el origen de la causa. Sin embargo, en ambos casos, el tribunal consideró que se cumplían del mismo modo los requisitos, dado que los Museos demandados realizaban una actividad comercial suficiente en el territorio que les permitía considerar que se comportaban como particulares ${ }^{30}$.

29 En la Sentencia de 8 de septiembre de 2009, del Tribunal de apelaciones, Noveno circuito, Claude Cassirer v. Kingdom of Spain, a Foreign State, se precisan las actividades que ameritan el reconocimiento de su actividad comercial en territorio de Estados Unidos y que permiten la aplicación de la excepción de expropiación. En el caso Altmann, incluso se aplicó el FSIA con carácter retroactivo. Ver en este sentido M. Goodman, “The Destruction of International Notions of Power and Sovereignty: The Supreme Court's Misguided Application of Retroactivity Doctrine to the Foreign Sovereign Immunities Act in Republic of Austria v. Altmann", The Georgetown Law Journal, 93 (2005): 1118. También en M.J. Chorazak, "Clarity and Confusion: Did Republic of Austria v. Altmann Revive State Department Suggestions of Foreign Sovereign Immunity?", Duke Law Journal, 55 (2005): 373 y ss.

30 En el caso Altmann, la Galería Belvedere por cuanto "authoring, editing, and publishing in the United States both a book entitled Klimt's Women, as well as an English-language guidebook, containing photographs of the looted paintings. (...) that the advertisements in the United States of Gallery exhibitions, particularly those relating to the Klimt paintings, as well as operation of the Gallery itself, constitute commercial activity. The key commercial behavior of the Gallery here is not its operation of the museum exhibition in Austria, however, but its publication and marketing of that exhibition and the 
Las consecuencias actuales de la privación ilícita de obras de arte en tiempos del nazismo Soledad Torrecuadrada García-Lozano

En conclusión, de conformidad con la Ley estadounidense, España no puede gozar de inmunidad porque el origen de la diferencia (la obra de arte cuya propiedad se discute) se encuentra en una adquisición ilícita - por parte de otro Estado- desde la perspectiva del Derecho Internacional. Llama la atención que el Derecho Internacional solo se tenga en cuenta en este punto y no en los restantes que rodean el caso.

\subsection{La inmunidad de ejecución}

En este subepígrafe me detendré brevemente en la inmunidad de ejecución de la que gozan los bienes del Estado de conformidad con el Derecho Internacional Público y, más concretamente con la Convención de Naciones Unidas sobre las inmunidades de los Estados y de sus bienes de 2004, máxime cuando se encuentran en el territorio del Estado demandado (ilustrando esta cuestión con el caso Cassirer), además de recordar el pronunciamiento de la Corte Internacional de Justicia en el caso de las inmunidades jurisdiccionales del Estado (Alemania c. Italia) ${ }^{31}$, referido precisamente al aspecto que ahora nos ocupa.

Teniendo en cuenta las disposiciones de la Convención de Naciones Unidas sobre las inmunidades de los Estados y de sus bienes de 2004, parece evidente que las obras de arte que puedan ser reclamadas ante los tribunales de un Estado distinto de aquel en el que se encuentra el bien en cuestión gozan, de conformidad con la Convención de 2004, de inmunidad de ejecución. Así, el artículo 19 del citado texto convencional es claro en este punto cuando se refiere a la "inmunidad del Estado respecto de medidas coercitivas posteriores al fallo", establece la imposibilidad de su adopción salvo: a) consentimiento expreso del Estado para ello; b) si el Estado ha "asignado o destinado bienes a la satisfacción de la demanda objeto de ese proceso"; o c) cuando se trata de bienes en el territorio del foro que se utilizan para fines distintos de los

oficiales no comerciales y sólo en el supuesto en el que "tengan un nexo con la entidad contra la cual se haya incoado el proceso". Es evidente que el cuadro reclamado no se encuentra en el territorio del foro, pues se mantiene en España, pero aunque así no fuera, la interpretación de

books in the United States. Klimt's Women, for example, is published in English in the United States by Yale University Press and capitalizes on the images of three of the paintings at issue. That book was published in conjunction with a large exhibition at the Gallery featuring the expropriated paintings. Furthermore, the Austrian Gallery asserts copyright ownership as 'authors'; two employees of the Gallery edited the book; and the director of the Gallery is listed as responsible for its content. The museum guidebook is also published in English and features the painting Adele Bloch-Bauer $I$ on its cover. The publication and sale of these materials and the marketing of the Klimt exhibition in the United States are commercial activities in and of themselves, but are also a means to attract American tourists to the Gallery. Given that the commercial activity is centered around the very paintings at issue in this action and far exceeds that which we found sufficient to justify applying \1605(a)(3)” Vid. En Sentencia citada supra en nota $\mathrm{n}^{\circ} 27$.

31 Vid. toda la documentación referida al caso en cuestión en Cour Internationale de Justice, "Immunités juridictionnelles de l'État (Allemagne c. Italie ; Grèce (intervenant)", http://www.icj-cij.org/fr/affaire/143 [consulta 30.jul.2018].

Número de página no utilizable para citar 
los fines aludidos ha de interpretarse de conformidad con el artículo 21 del mismo texto que nos indica los bienes que no pertenecen a la categoría indicada en el apartado c), entre los que figuran:

“d) los bienes que formen parte del patrimonio cultural del Estado, o parte de sus archivos, y no se hayan puesto ni estén destinados a ser puestos en venta; e) los bienes que formen parte de una exposición de objetos de interés científico, cultural o histórico y no se hayan puesto ni estén destinados a ser puestos en venta".

Por tanto, en la medida en que la obra reclamada inicialmente por Claude Cassirer (y, tras su fallecimiento, por sus herederos) no está puesta a la venta ni destinada a ello ni se encuentra en el territorio del foro (con lo que al ubicarse en el territorio del demandado goza, cuando menos, de la misma protección, pues el Derecho Internacional solo limita la inmunidad de ejecución de los bienes situados en el territorio del Estado del foro), sería imprescindible la cooperación por parte de España mediante una entrega voluntaria, en el supuesto de fallar en este sentido los tribunales estadounidenses, para que ello resultara acorde al Derecho Internacional. En todo caso, observemos en este punto que, de conformidad con la Ley española, para la exportación de una obra del patrimonio histórico español se requiere la autorización expresa de la administración del Estado para su exportación ${ }^{32}$, que no dudo se obtendría si el tribunal estadounidense reclamara la pintura, pero sería una muestra de buena voluntad por parte de las autoridades, pues jurídicamente el bien en cuestión goza de protección.

Esta situación nos conduce a la Sentencia de la Corte Internacional de Justicia antes enunciada, que objetivamente cuenta con similitudes más que relevantes con el caso en presencia, pues se trata sobre la inmunidad de ejecución de Villa Vigoni, donde se ubica el Centro italo-alemán que promueve las relaciones entre ambos Estados en perspectiva europea e internacional. Se trata, por tanto, de un bien de naturaleza cultural, aunque inmueble.

Los hechos que fundamentaron la demanda tienen que ver con el caso Cassirer relativamente, pero considero relevante recordar este asunto porque de él podemos extraer alguna enseñanza del órgano judicial principal de las Naciones Unidas, en un supuesto en el que se intentaba equilibrar de un lado la inmunidad del Estado alemán y en el otro la vulneración de normas imperativas, categoría dentro de la que se encuentran los hechos acontecidos en la Segunda Guerra Mundial. En este caso eran personas de nacionalidad italiana deportadas entre 1943 y 1945 para trabajar de forma forzosa al servicio del Reich que no habían recibido ningún tipo de reparación por ello, a pesar de la Ley Federal alemana relativa a la indemnización de víctimas de la persecución nacionalsocialista de 1953. Numerosos nacionales italianos no pudieron obtener esa indemnización debido, en su mayoría, a que carecían de residencia o domicilio permanente

32 Se trata del artículo 5 de la Ley 16/1985, de 25 de junio, de Patrimonio Histórico español, https://www.boe.es/buscar/act.php?id=BOE-A-1985-12534 [consulta 30.jul.2018]. 
Las consecuencias actuales de la privación ilícita de obras de arte en tiempos del nazismo Soledad Torrecuadrada García-Lozano

en Alemania, requisito para ello en virtud de la Ley $^{33}$. Alemania demandó a Italia por entender vulnerada su inmunidad de jurisdicción debido a las decisiones de los jueces italianos adoptando medidas de ejecución forzosa contra Villa Vigoni, propiedad del Estado alemán en territorio italiano, en respuesta las demandas presentadas por sus nacionales víctimas de los hechos indicados ${ }^{34}$.

La Corte Internacional de Justicia, entendió en este caso la prevalencia de la inmunidad de jurisdicción del Estado extranjero incluso para reparar la vulneración de normas imperativas. La Corte fundamentó esta decisión en algo que comentamos antes: la imposibilidad de adoptar lícitamente una medida de embargo contra un bien cultural (Villa Vigoni es un centro cultural). A ello se añade la ausencia de consentimiento en ello por parte de Alemania, motivo por el cual prevalece la inmunidad de ejecución del Estado. Así las cosas, la inscripción de una hipoteca judicial sobre esta propiedad constituye una violación por Italia de su obligación de respetar la inmunidad debida a Alemania.

Es evidente que el razonamiento de la Corte Internacional de Justicia en el caso de Alemania c. Italia favorecería la inmunidad de ejecución de los Estados reclamados en este punto (como sería el caso de España), propietario de la obra cuya restitución se solicita, tanto en su vertiente de jurisdicción como de ejecución.

\section{Conclusiones}

Una de las críticas que se hace a España es que ha participado en la adopción de todas las declaraciones en las que incluyen decisiones respecto de la devolución de los bienes confiscados durante el nazismo (Washington, Viena, Terezín, etc.). Sin embargo, no es menos cierto que lo que allí se asumieron fueron compromisos de naturaleza política, que no resultan jurídicamente exigibles, como ya nos indicó algún tribunal estadounidense en ocasiones anteriores. Estos textos muestran la voluntad estatal de resolver un problema histórico, incluso, si fuera preciso, modificando los ordenamientos internos para superar las dificultades que puedan presentar en lo que se refiere a la restitución de los bienes que se encuentren en su territorio. En España no se han adoptado este tipo de modificaciones, pero no podemos ignorar en este punto que ha

33 Como interviniente en el supuesto participó Grecia debido a que el 10 de junio de 1944, durante la ocupación alemana, sus fuerzas armadas perpetraron una masacre en Distomo, muriendo múltiples civiles, cuyos causahabientes acudieron en 1995 al Tribunal de Primera Instancia de Livadia una acción por daños y perjuicios contra Alemania para procurar una reparación por las pérdidas humanas y materiales sufridas.

34 Los hechos que figuran en este párrafo son una síntesis de la Sentencia de la Corte, de 3 de febrero de 2012, en el asunto de las Inmunidades jurisdiccionales del Estado (Alemania c. Italia); con Grecia como interviniente. La versión auténtica de la sentencia se encuentra en http://www.icj-cij.org/fr/affaire/143/arrets\&gws_rd=cr\&dcr=0\&ei=JDMWuXsCcOyUZKnieAD [consulta 30.jul.2018].

Número de página no utilizable para citar 
habido dos casos de bienes de estas características (el otro se resolvió mediante negociación entre los interesados), por lo que el Estado español se encuentra muy alejado de otros Estados de su entorno en este punto. A pesar de lo cual, España debería cumplir las obligaciones que se ha autoimpuesto, por mucho que cuenten con naturaleza política. Por ello sería positivo que se adoptaran los procedimientos de derecho interno aplicables si se llegara a plantear otro supuesto de estas características.

En relación con las excepciones a la inmunidad de jurisdicción del Estado extranjero hay que tener en cuenta como punto de partida que los tribunales nacionales han de respetar esta excepción procesal en los justos términos definidos por el Derecho Internacional. En este sentido, es evidente que la excepción aplicada por los tribunales estadounidenses se excede a lo internacionalmente establecido. Es cierto que la Convención de Naciones Unidas sobre las inmunidades de los Estados y de sus bienes de 2004 aún no ha entrado en vigor (cuenta a día de hoy con 21 Estados parte de los 30 exigidos para su entrada en vigor ${ }^{35}$ ) y que Estados Unidos no se encuentra entre ellos, pero en la medida en que una parte muy relevante de sus disposiciones se ha consolidado como norma consuetudinaria ${ }^{36}$ cabría plantearse si Estados Unidos al actuar de este modo ha vulnerado una norma internacional, o podríamos encontrarnos ante actos modificativos de una costumbre internacional. En todo caso, la realidad actual nos ubica ante el primer supuesto recién planteado.

Es cierto que debemos plantearnos la excepción a la inmunidad de jurisdicción del Estado debido a vulneraciones graves del Derecho Internacional, inexistente en estos momentos en la Convención de Naciones Unidas sobre las inmunidades de los Estados y de sus bienes de 2004. Pero, aunque se incorporase esta nueva excepción no afectaría al caso en presencia, por cuanto permitiría el ejercicio de jurisdicción sobre el autor de los hechos calificables como vulneración grave del Derecho Internacional, lo que no siempre resultará un fundamento jurídico suficiente para su aplicación a la restitución de las obras de las que se privó ilícitamente a sus propietarios en la época del horror nazi.

A la vista de las dificultades en presencia, debería subrayarse la conveniencia de acudir a los tribunales en cuyo territorio se encuentra el bien en cuestión, especialmente si se trata de una propiedad estatal, pues de este modo las víctimas o sus herederos superarían los inconvenientes derivados de las inmunidades de las que son titulares los Estados.

35 El estado actual de la Convención a este respecto se puede consultar en https://treaties.un.org/Pages/ViewDetails.aspx?src=IND\&mtdsg_no=III-13\&chapter=3\&lang=en [consulta 30.jul.2018].

36 El carácter consuetudinario de estas normas no solo se afirma en el preámbulo de la convención, sino que deriva de la práctica generalizada de los Estados. Además, su contenido sustantivo ha sido reconocido como norma consuetudinaria de Derecho Internacional también por tribunales nacionales, como el Tribunal Constitucional Español (véase, entre otras, la STC 107/92), e internacionales (como en el ya citado fallo de la Corte Internacional de Justicia en el caso de Alemania c. Italia). 
Las consecuencias actuales de la privación ilícita de obras de arte en tiempos del nazismo Soledad Torrecuadrada García-Lozano

Una enseñanza clara que evidencia este caso es que cuando uno acude a los tribunales a defender una posición, no puede pretender, como afirmó Ronald Lauder, Presidente del Congreso Mundial Judío que se responda atendiendo a criterios morales y no a los jurídicos. Los tribunales son órganos de naturaleza jurisdiccional que han de actuar aplicando normas jurídicas y no morales. En consecuencia, estoy firmemente convencida de que este no es un escenario en el que se puedan hacer valer criterios morales, máxime si quien pretende hacerlos valer es quien acude a un tribunal. Tampoco creo que sea admisible la criminalización de un Estado que ha sido demandado ante un tribunal de otro y pretende defender sus intereses en presencia. Cuestionar si "¿fue España una Arcadia para el expolio nazi?”37, con el único fundamento del caso Cassirer, no es solo exagerado, por cuanto este cuadro llegó a España en las últimas décadas del siglo XX, después de haber pasado los años de la posguerra y hasta entonces en territorio estadounidense. Quizá otros supuestos permitan esta afirmación, pero éste precisamente no parece un fundamento serio para una pregunta de estas características

En la actualidad vivimos en un mundo en el que es más importante la apariencia que la razón y, si bien el Barón Thyssen-Bornemisza nos proporcionó un museo maravilloso en el centro de Madrid, las historias recientemente aparecidas en la prensa, junto con otras ya previamente conocidas respecto de las relaciones pasadas de la familia con el Nazismo, consiguen opacar las circunstancias del caso concreto, en una interpretación muy fácil de realizar sin necesidad de profundizar en el contexto que rodea a la obra de Cassirer. También hemos de observar que, el Barón adquirió en 1976 el cuadro en Estados Unidos y que siempre estuvo expuesto al público desde entonces, primero en Villa Favorita (Lugano, Suiza) y después, cuando se constituyó la Fundación Colección Thyssen-Bornemisza, en el Museo que lleva este nombre en Madrid.

Como algunos autores han sostenido, la cuestión de la restitución de los bienes incautados por el nazismo afecta de modo muy sensible a las relaciones internacionales, elevando cuestiones cruciales sobre la separación de poderes y la discrecionalidad judicial ${ }^{38}$. Otro espacio sensible sobre el que se podría actuar de cara a evitar problemas similares en el futuro es el del mercado del arte, caracterizado por la flexibilidad y la falta de investigación (en muchas ocasiones de forma interesada) en aras a la obtención de un mayor rendimiento. El control de este mercado de forma real por los ordenamientos nacionales (más allá de códigos de conducta, con un cumplimiento difícilmente exigible) procuraría una mayor confiabilidad en un sector que tradicionalmente ha hecho gala de escasos escrúpulos en lo que a la limpieza de las transmisiones y el origen de las piezas que ofrece se refiere.

37 Vid en: M.A. García Vega, “España cuna arcadia para el expolio nazi??”, El País, http://elpais.com/elpais/2015/01/12/eps/1421064647_501434.html [consulta 30.jul.2018].

38 El texto transcrito se encuentra en E. Wolf, "The Ninth Circutit's Decision in Von Saher v. Norton Simon Museum of Art at Pasadena: The Invocation of the Art of State Doctrine and Its Implications for Future Nazi-Stolen Art Claims", Cardozo Arts \& Entertainment Law Journal, 34 (2016): 553, aunque la reflexión subyacente puede leerse en: N. O’Donnell, "Restitution Claims for Cranach Paintings in the Norton Simon Museum Revived by Ninth Circuit, Case How Hinges on Act of State Doctrine", Art Law Report, 2014 o en Arp, "Dos males, un bien no hacen". 
Vista la dispersión de soluciones aplicadas a los casos en función de los tribunales competentes y del derecho aplicable a cada uno de ellos, quizá haya llegado el momento de plantear un procedimiento para procurar que las soluciones a todos estos casos resulten lo más homogéneas posibles y no tengan exclusivamente en cuenta la reparación económica (si procediera) sino también la reparación moral ${ }^{39}$. Esta no es una idea novedosa, bien al contrario, ya la lanzó a finales del siglo XX Owen C. Pell ${ }^{40}$, proponiendo la creación de una comisión de mediación o arbitraje a estos efectos. Comisión que debería tener como instrumento constitutivo un tratado internacional con amplia participación y que aplicaría reglas uniformes para este tipo de casos, siempre atendiendo evidentemente a las circunstancias concretas que rodean cada uno de los supuestos en presencia. J. A. Kreder, por su parte, sugiere la creación de un tribunal internacional con este propósito ${ }^{41}$. Claro que la existencia de una institución independiente, como propone la autora, puede no provocar demasiadas adhesiones entre los Estados, siempre desconfiados de esa independencia. No obstante, sí que es cierto que el establecimiento de un órgano encargado de resolver estos conflictos sería muy útil porque serviría para que las soluciones aplicables en cada uno de los casos no se hicieran depender del tribunal competente ni del derecho nacional aplicable, con grandes diferencias entre los sistemas jurídicos, sin que ello pueda entenderse en el sentido de que una perspectiva venza a la otra, sino como la búsqueda de un punto medio razonable a los efectos que se persiguen.

39 Sobre este aspecto (daños morales en relación con el Holocausto) puede verse la Sentencia de 11 de noviembre de 1991, $\mathrm{n}^{\circ}$ 214/1991, de la Sala Primera del Tribunal Constitucional y el magnífico estudio que realiza G. Minero Alejandre, La protección post mortem de los derechos al honor, intimidad y propia imagen y la tutela frente al uso de datos de carácter personal tras el fallecimiento, (Madrid, Aranzadi, 2018).

40 O.C. Pell, "The Potential for a Mediation/Arbitration Commission to Resolve Disputes Relating to Artworks Stolen or Looted during World War II”, DePaul Journal of Art, Technology \& Intellectual Property Law, 10 (1999): 27 y ss.

41 J.A. Kreder, "Reconciling Individual and Group Justice with the Need for Repose in Nazi-Looted Art Disputes - Creation of an International Tribunal”, Brooklyn Law Review, 73 (2007): 155 y ss.

Número de página no utilizable para citar 